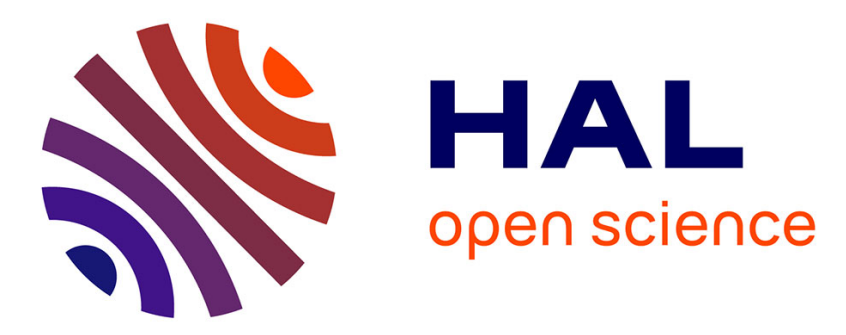

\title{
On the bending of viscoelastic plates made of polymer foams
}

\author{
Holm Altenbach, Victor A. Eremeyev
}

\section{To cite this version:}

Holm Altenbach, Victor A. Eremeyev. On the bending of viscoelastic plates made of polymer foams. Acta Mechanica, 2009, 204 (3-4), pp.137-154. hal-00821487

\section{HAL Id: hal-00821487 \\ https://hal.science/hal-00821487}

Submitted on 21 May 2013

HAL is a multi-disciplinary open access archive for the deposit and dissemination of scientific research documents, whether they are published or not. The documents may come from teaching and research institutions in France or abroad, or from public or private research centers.
L'archive ouverte pluridisciplinaire HAL, est destinée au dépôt et à la diffusion de documents scientifiques de niveau recherche, publiés ou non, émanant des établissements d'enseignement et de recherche français ou étrangers, des laboratoires publics ou privés. 


\section{On the bending of viscoelastic plates made of polymer foams}

Abstract Considering the viscoelastic behavior of polymer foams a new plate theory based on the direct approach is introduced and applied to plates composed of functionally graded materials (FGM). The governing two-dimensional equations are formulated for a deformable surface, the viscoelastic stiffness parameters are identified assuming linear-viscoelastic material behavior. The material properties are changing in the thickness direction. Solving some problems of the global structural analysis it will be demonstrated that in some cases the results significantly differ from the results based on the Kirchhoff-type theory.

\section{Introduction}

Foams are a very perspective class of materials for modern engineering applications. Metallic and polymeric foams are more and more used as materials for lightweight structures [9,10,14,16,23-25]. Such structures are applied in civil engineering, in the automotive or airspace industries since they combine low weight, high specific strength, and excellent possibilities to absorb energy. The technical realization is mostly performed as sandwich panels (plates or shells with hard and stiff face sheets and a core layer made of a foam). From the mechanical point of view, a foam is a very complex material which has many non-classical properties (for example, some foams have a negative Poisson's ratio, see $[16,20,34]$ for details). The non-homogeneous foam itself can be modeled as a functionally graded material (FGM) with "smeared" mechanical properties changing over the thickness direction. There are two types of metal or polymer foams. One is the closed-cell foam, while the second one is the open-cell foam. The defining characteristic of the foams is the very high porosity: typically well over 80, 90 and even $98 \%$ of the volume consists of void spaces. Examples of polymer open-cell and closed-cell foams are presented in Fig. 1.

The analysis of plates and shell structures can be performed on the base of theories deduced by various approaches. Finally, all theories are presented by two-dimensional field equations. Mostly engineering hypotheses about the stress, strain or displacement states in the plate are applied, and one gets some simplifications of the basic three-dimensional equations. They result in two-dimensional equations connecting the kinematics with the strains, stating the static equilibria or equations of motion and founding the constitutive relations. In a similar way applying some mathematical techniques like the power series expansion or the asymptotic 


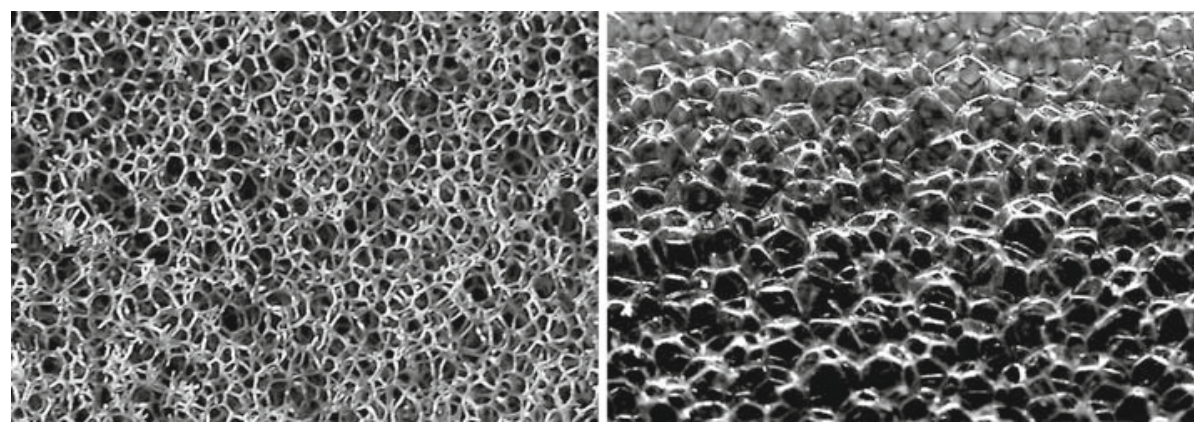

Fig. 1 Polymer open-cell (left) and closed-cell (right) foams
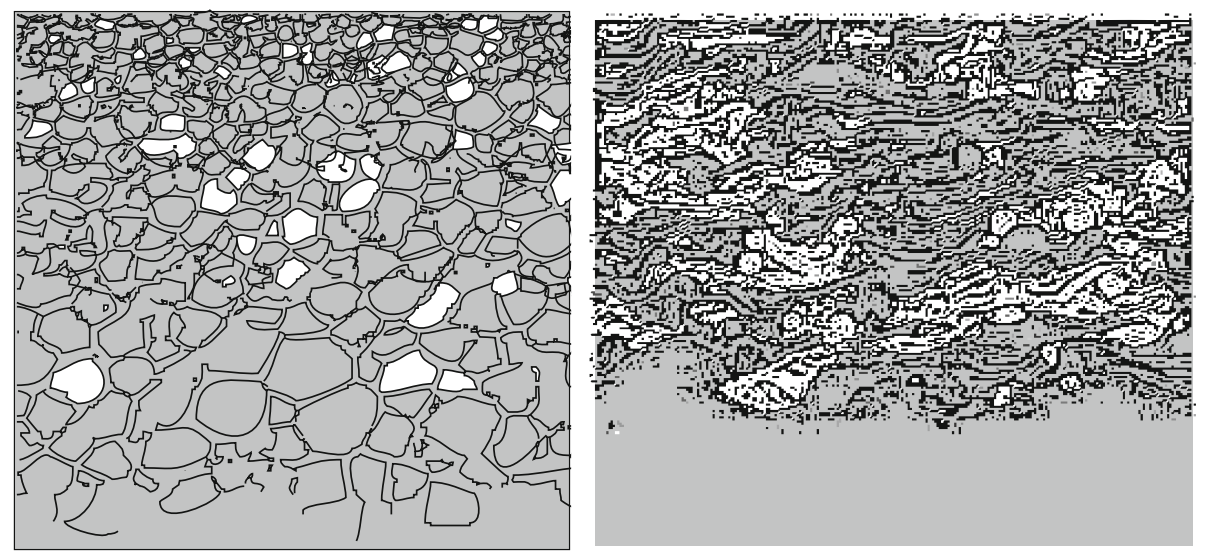

Fig. 2 Non-homogeneous structures: foam (left), thermal coating (right)

integration one can observe the set of two-dimensional governing equations. Note that both approaches have a unique starting point- the equations for the three-dimensional continuum. In contrast, the direct approach is based on the straight-forward introduction of two-dimensional equations without any a priori three-dimensional assumptions. This approach in combination with the effective properties concept allows the global analysis in all branches of the plate theory (homogeneous, sandwich, laminated, etc.). The different possibilities of the formulation of plate theories are discussed in [27,31] among others.

Here we present a new theory of viscoelastic plates with changing properties in the thickness direction based on the direct approach in the plate theory and added by the effective properties concept. We consider plates made of polymer foams with highly non-homogeneous structure through the thickness (see, for example Fig. 2). We apply the theory of plates and shells formulated earlier in [1-3,38,39]. A similar approach was suggested in [33], but homogeneous material behavior was assumed. From the direct approach point of view a plate or a shell is modeled as a material surface each particle of which has five degrees of freedom (three displacements and two rotations, the rotation about the normal to the plate is not considered as a kinematically independent variable). Such a model can be accepted in the case of plates with constant or slowly changing thickness. For the linear variant the identification of the elastic stiffness tensors considering changing properties was proposed in [4-6]. Using the techniques presented in these articles the static boundary-value problems for FGM plates made of metal foams which behave elastically are solved in [7]. Some extensions of the proposed theory of plates to the case of viscoelastic polymer foams were given in [8].

\section{Governing equations}

Let us consider for brevity the geometrically and physically linear theory. In addition, we assume plate-like structures. The basic equations interlinking the strains with the displacements and rotations or stating the equilibrium (static or dynamic) can be deduced applying hypotheses (like the Kirchhoff's hypotheses) or mathematical techniques (like power series expansion). In both cases one gets automatically the expressions for the constitutive behavior assuming elastic or inelastic material behavior. 
A quite different way is given by the direct approach. The starting point in this case is a two-dimensional deformable surface. On each part of this deformable surface forces and moments are acting - they are the primary variables. The next step is the introduction of the deformation measures. Finally, it is necessary to combine the forces and the moments with the deformation variables (constitutive equations). In comparison with the other approaches such a theory is formulated in a more natural way. But the identification of the effective properties (stiffness and other parameters) must be realized for each class of plates individually solving, for example, boundary value problems. The identification of the two-dimensional characteristics is a non-trivial problem since they must be computed from the three-dimensional parameters applying assumptions like the introduction of stress resultants (forces and moments) instead the stress tensor components.

\subsection{Symbolic representation}

Let us introduce the governing equations. The equations of motion are formulated as Euler's laws of dynamics [5-7],

$$
\nabla \cdot \boldsymbol{T}+\boldsymbol{q}=\rho \ddot{u}+\rho \Theta_{1} \cdot \ddot{\boldsymbol{\varphi}}, \quad \boldsymbol{\nabla} \cdot \boldsymbol{M}+\boldsymbol{T}_{\times}+\boldsymbol{m}=\rho \Theta_{1}^{\mathrm{T}} \cdot \ddot{\boldsymbol{u}}+\rho \Theta_{2} \cdot \ddot{\varphi} .
$$

Here $\boldsymbol{T}, \boldsymbol{M}$ are the tensors of forces and moments, $\boldsymbol{q}, \boldsymbol{m}$ are the vectors of surface loads (forces and moments), $T_{\times}$is the vector invariant of the force tensor, $\nabla$ is the nabla (Hamilton) operator, $u, \varphi$ are the vectors of displacements and rotations, $\Theta_{1}, \Theta_{2}$ are the first and the second tensor of inertia, $\rho$ is the density (effective property of the deformable surface) $(\ldots)^{\mathrm{T}}$ denotes transposed and $(\ldots)$ the time derivative. The geometrical equations are given as

$$
\boldsymbol{\mu}=(\nabla \boldsymbol{u} \cdot \boldsymbol{a})^{\mathrm{sym}}, \quad \gamma=\nabla \boldsymbol{u} \cdot \boldsymbol{n}+\boldsymbol{c} \cdot \boldsymbol{\varphi}, \quad \boldsymbol{\kappa}=\nabla \varphi .
$$

$a$ is the first metric tensor (plane tensor), $\boldsymbol{n}$ is the unit outer normal vector at the surface, $c$ is the discriminant tensor $(c=-a \times n), \mu, \gamma$ and $\kappa$ are the strain tensors (the tensor of in-plane strains, the vector of transverse shear strains and the tensor of the out-of-plane strains), (... $)^{\text {sym }}$ denotes the symmetric part.

The boundary conditions are given by the relations

$$
v \cdot T=f, \quad v \cdot M=l \quad(l \cdot n=0) \text { or } u=u^{0}, \quad \varphi=\varphi^{0} \text { along } S .
$$

Here $f$ and $l$ are external force and moment vectors acting along the boundary of the plate $S$, while $\boldsymbol{u}^{0}$ and $\varphi^{0}$ are given functions describing the displacements and rotations of the plate boundary, respectively. $v$ is the unit outer normal vector to the boundary $S(v \cdot n=0)$. The relations (2) are the static and the kinematic boundary conditions. Other types of boundary conditions are possible. For example, the boundary conditions corresponding to a hinge are given by

$$
\nu \cdot M \cdot \tau=0, \quad u=0, \quad \varphi \cdot \tau=0 .
$$

Here $\tau$ is the unit tangent vector in the tangential plane to the boundary $S(\tau \cdot n=\tau \cdot v=0)$.

\subsection{Basic equations in Cartesian coordinates}

Let us assume the Cartesian coordinate system $x_{1}, x_{2}$ (in-plane coordinates) and $z$ (orthogonal to the midplane). Then the unit normal vectors are $\boldsymbol{e}_{1}, \boldsymbol{e}_{2}$ and $\boldsymbol{n}$. With respect to the introduced coordinate system the following representations are valid:

- Displacement and rotation vectors:

$$
u=u_{1} \boldsymbol{e}_{1}+u_{2} e_{2}+w n, \quad \varphi=-\varphi_{2} \boldsymbol{e}_{1}+\varphi_{1} e_{2} .
$$

$u_{\alpha}(\alpha=1,2)$ are the in-plane displacements, $w$ is the deflection and $\varphi_{\alpha}$ are the rotations about the axes $e_{\alpha}$, respectively.

- Force and moment tensors:

$$
\begin{aligned}
& T=T_{1} \boldsymbol{e}_{1} \boldsymbol{e}_{1}+T_{12}\left(\boldsymbol{e}_{1} e_{2}+\boldsymbol{e}_{2} \boldsymbol{e}_{1}\right)+T_{2} \boldsymbol{e}_{2} e_{2}+T_{1 n} \boldsymbol{e}_{1} \boldsymbol{n}+T_{2 n} \boldsymbol{e}_{2} \boldsymbol{n} \\
& \boldsymbol{M}=M_{1} e_{1} \boldsymbol{e}_{2}-M_{12}\left(e_{1} e_{1}-e_{2} e_{2}\right)-M_{2} e_{2} e_{1}
\end{aligned}
$$

$T_{\alpha}, T_{12}$ are the in-plane forces, $T_{\alpha n}$ are the transverse shear forces, $M_{\alpha}$ are the bending moments and $M_{12}$ is the torsion moment. 
- Strain tensors:

$$
\begin{aligned}
& \mu=\mu_{1} \boldsymbol{e}_{1} \boldsymbol{e}_{1}+\mu_{12}\left(e_{1} \boldsymbol{e}_{2}+\boldsymbol{e}_{2} e_{1}\right)+\mu_{2} \boldsymbol{e}_{2} \boldsymbol{e}_{2}, \\
& \gamma=\gamma_{1} \boldsymbol{e}_{1}+\gamma_{2} \boldsymbol{e}_{2} \\
& \kappa=\kappa_{1} \boldsymbol{e}_{1} \boldsymbol{e}_{2}-\kappa_{12} \boldsymbol{e}_{1} \boldsymbol{e}_{1}+\kappa_{21} \boldsymbol{e}_{2} \boldsymbol{e}_{2}-\kappa_{2} \boldsymbol{e}_{2} \boldsymbol{e}_{1} .
\end{aligned}
$$

$\mu_{k}$ are the strains, $\mu_{12}$ is the shear strain, $\gamma_{k}$ are the transverse shear strains, $\kappa_{k}$ are the bending deformations and $\kappa_{12}$ is the torsion deformation.

- External loads:

$$
\boldsymbol{q}=q_{1} \boldsymbol{e}_{1}+q_{2} \boldsymbol{e}_{2}+q_{n} n, \quad m=-m_{2} e_{1}+m_{1} e_{2} .
$$

$q_{k}$ are the in-plane loads, $q_{n}$ is the transverse load, $m_{k}$ are the moments.

Now the first and the second Euler's law and the geometrical relations take the form (quasi-static case):

- First and second Euler's law:

$$
\begin{aligned}
T_{1,1}+T_{12,2}+q_{1} & =0, \quad T_{12,1}+T_{2,2}+q_{2}=0, \\
T_{1 n, 1} & +T_{2 n, 2}+q_{n}=0, \\
M_{1,1}+M_{12,2}-T_{1 n}+m_{1} & =0, \quad M_{12,1}+M_{2,2}-T_{2 n}+m_{2}=0 .
\end{aligned}
$$

- Boundary conditions (for brevity, we present it when $S$ is a part of line $x_{1}=$ const $\left(v=e_{1}, \tau=e_{2}\right)$ ):

- Static boundary conditions

$$
T_{1}=f_{1}, \quad T_{12}=f_{2}, \quad M_{1}=l_{1}, \quad M_{12}=l_{2}
$$

- Kinematic boundary conditions

$$
u_{1}=u_{1}^{0}, \quad u_{2}=u_{2}^{0}, \quad w=w^{0} \quad \varphi_{1}=\varphi_{1}^{0}, \quad \varphi_{2}=\varphi_{2}^{0},
$$

- Boundary conditions for a hinge

$$
M_{1}=0, \quad u_{1}=u_{2}=w=0 \quad \varphi_{2}=0 .
$$

- Geometrical relations:

$$
\begin{aligned}
& \mu_{1}=u_{1,1}, \quad \mu_{2}=u_{2,2}, \quad \mu_{12}=\frac{1}{2}\left(u_{1,2}+u_{2,1}\right), \\
& \gamma_{1}=w_{, 1}+\varphi_{1}, \quad \gamma_{2}=w_{, 2}+\varphi_{2}, \\
& \kappa_{1}=\varphi_{1,1}, \quad \kappa_{2}=\varphi_{2,2}, \quad \kappa_{12}=\varphi_{2,1}, \quad \kappa_{21}=\varphi_{1,2} .
\end{aligned}
$$

\section{Constitutive equations}

Polymers near their glass transition temperature behave like viscoelastic materials [11,23-25,32]. That means that the moduli of the polymers depend on the strain-rate or the time of loading. Thus, a foam made of such a polymer behaves viscoelastically, too. Experimental investigations of the vicoelasticity of foams were presented in $[16,19]$. The two-dimensional constitutive equations of a viscoelastic plate were formulated in the general form in [2]. A through-the-thickness symmetric structure of the plate and an isotropic material behavior was considered in [8]. Here we consider the general anisotropic case of two-dimensional viscoelastic constitutive equations. In this case there follow the constitutive equations for the stress resultants:

- In-plane forces:

$$
\begin{aligned}
\boldsymbol{T} \cdot \boldsymbol{a} & =\mathcal{A} \boldsymbol{\mu}+\mathcal{B} \boldsymbol{\kappa}+\tilde{\mathcal{G}}_{1} \boldsymbol{\gamma} \\
& \equiv \int_{-\infty}^{t} \boldsymbol{A}(t-\tau) \cdot \dot{\mu}(\tau) \mathrm{d} \tau+\int_{-\infty}^{t} \boldsymbol{B}(t-\tau) \cdot \dot{\boldsymbol{\kappa}}(\tau) \mathrm{d} \tau+\int_{-\infty}^{t} \dot{\gamma}(\tau) \cdot \Gamma_{1}(t-\tau) \mathrm{d} \tau,
\end{aligned}
$$


- Transverse shear forces:

$$
\begin{aligned}
T \cdot n & =\mathcal{G} \gamma+\mathcal{G}_{1} \boldsymbol{\mu}+\mathcal{G}_{2} \kappa \\
& \equiv \int_{-\infty}^{t} \Gamma(t-\tau) \cdot \dot{\gamma}(\tau) \mathrm{d} \tau+\int_{-\infty}^{t} \Gamma_{1}(t-\tau) \cdot \dot{\mu}(\tau) \mathrm{d} \tau+\int_{-\infty}^{t} \Gamma_{2}(t-\tau) \cdot \cdot \dot{\kappa}(\tau) \mathrm{d} \tau,
\end{aligned}
$$

- Moments:

$$
\begin{aligned}
M^{\mathrm{T}} & =\tilde{\mathcal{B}} \mu+\mathcal{C} \boldsymbol{\kappa}+\tilde{\mathcal{G}}_{2} \gamma \\
& \equiv \int_{-\infty}^{t} \dot{\mu}(\tau) \cdot \boldsymbol{B}(t-\tau) \mathrm{d} \tau+\int_{-\infty}^{t} \boldsymbol{C}(t-\tau) \cdot \dot{\kappa}(\tau) \mathrm{d} \tau \int_{-\infty}^{t} \dot{\gamma}(\tau) \cdot \Gamma_{2}(t-\tau) \mathrm{d} \tau .
\end{aligned}
$$

Here $\mathcal{A}, \mathcal{B}, \tilde{\mathcal{B}}, \mathcal{C}, \mathcal{G}, \mathcal{G}_{1}, \mathcal{G}_{2}, \tilde{\mathcal{G}}_{1}, \tilde{\mathcal{G}}_{2}$, are linear viscoelastic operators, $A(t), B(t), C(t)$ are fourth rank tensors, $\Gamma_{1}(t), \Gamma_{2}(t)$ are third rank tensors, $\Gamma(t)$ is a second rank tensor which describe the effective stiffness properties (relaxation functions for the plate). They depend on the material properties and the cross-section geometry.

Using the assumptions that $A(t), B(t)$, etc., do not depend on time with the relations $\mu(-\infty)=0$, $\kappa(-\infty)=0, \gamma(-\infty)=0$ then from Eqs. (12)-(14) we obtain the constitutive equations of elastic plates presented in $[5-7,38]$.

Let us consider orthotropic material behavior and a plane mid-surface. In this case instead of the general form of the effective stiffness tensors one gets [5-7,38]

$$
\begin{aligned}
& A=A_{11} a_{1} a_{1}+A_{12}\left(a_{1} a_{2}+a_{2} a_{1}\right)+A_{22} a_{2} a_{2}+A_{44} a_{4} a_{4}, \\
& B=B_{13} \boldsymbol{a}_{1} \boldsymbol{a}_{3}+B_{14} a_{1} a_{4}+B_{23} \boldsymbol{a}_{2} \boldsymbol{a}_{3}+\boldsymbol{B}_{24} \boldsymbol{a}_{2} \boldsymbol{a}_{4}+B_{42} a_{4} a_{2}, \\
& C=C_{22} a_{2} a_{2}+C_{33} \boldsymbol{a}_{3} a_{3}+C_{34}\left(\boldsymbol{a}_{3} \boldsymbol{a}_{4}+a_{4} a_{3}\right)+C_{44} a_{4} a_{4}, \\
& \Gamma=\Gamma_{1} \boldsymbol{a}_{1}+\Gamma_{2} \boldsymbol{a}_{2}, \quad \Gamma_{1}=0, \quad \boldsymbol{\Gamma}_{2}=0
\end{aligned}
$$

with

$$
a_{1}=a=e_{1} e_{1}+e_{2} e_{2}, \quad a_{2}=e_{1} e_{1}-e_{2} e_{2}, \quad a_{3}=c=e_{1} e_{2}-e_{2} e_{1}, \quad a_{4}=e_{1} e_{2}+e_{2} e_{1}
$$

$\boldsymbol{e}_{1}, \boldsymbol{e}_{2}$ are unit basic vectors. In addition, one obtains the orthogonality condition for $\boldsymbol{a}_{i}$,

$$
\frac{1}{2} a_{i} \cdot a_{j}=\delta_{i j}, \quad \delta_{i j}=\left\{\begin{array}{ll}
1, & i=j, \\
0, & i \neq j,
\end{array} \quad(i=1,2,3,4),\right.
$$

where $\delta_{i j}$ is the Kronecker's symbol.

In the case of isotropic and symmetric over the thickness plates the effective stiffness tensors have the following structure $[5-7,38]$ :

$$
\boldsymbol{A}=A_{11} a_{1} a_{1}+A_{22}\left(a_{2} a_{2}+a_{4} a_{4}\right), \quad \boldsymbol{C}=C_{22}\left(a_{2} a_{2}+a_{4} a_{4}\right)+C_{33} a_{3} a_{3}, \quad \Gamma=\Gamma \boldsymbol{a} .
$$

\section{Effective properties}

For elastic plates the identification of the components of the effective stiffness tensors was proved in [5-7,38]. By the same technique below the analogous viscoelastic stiffness tensor components are computed. Let us consider the three-dimensional viscoelastic constitutive equations $[12,15,18]$

$$
\boldsymbol{\sigma}=\int_{-\infty}^{t} \boldsymbol{R}(t-\tau) \cdot \cdot \dot{\varepsilon}(\tau) \mathrm{d} \tau
$$

or

$$
\varepsilon=\int_{-\infty}^{t} J(t-\tau) \cdot \cdot \dot{\sigma} \mathrm{d} \tau,
$$


where $\sigma$ and $\varepsilon$ are the tensors of stress and strain, $R(t)$ and $J(t)$ are the fourth rank tensors of relaxation and creep functions, respectively.

For an isotropic viscoelastic material Eqs. (15), (16) reduce to

$$
\sigma=\int_{-\infty}^{t} R_{1}(t-\tau) \dot{\boldsymbol{e}}(\tau) \mathrm{d} \tau+\int_{-\infty}^{t} R_{2}(t-\tau) I \operatorname{tr} \dot{\varepsilon}(\tau) \mathrm{d} \tau
$$

or

$$
\varepsilon=\int_{-\infty}^{t} J_{1}(t-\tau) \dot{s} \mathrm{~d} \tau+\int_{-\infty}^{t} J_{2}(t-\tau) I \operatorname{tr} \dot{\sigma} \mathrm{d} \tau
$$

with two scalar relaxation functions $R_{1}$ and $R_{2}$ and two scalar creep functions $J_{1}$ and $J_{2}$ which describe the shear and bulk properties of a viscoelastic isotropic material. $\boldsymbol{I}$ is the three-dimensional unit tensor. In addition, $s$ and $e$ are the deviatoric parts of the stress and the strain tensors, respectively.

Further we consider two cases:

Case 1 Homogeneous plates-all properties are constant (no dependency of the thickness coordinate $z$ ).

Case 2 Inhomogeneous plates (sandwich, multilayered, functionally graded)—all properties are functions of $z$.

That means that in general $R$ and $\boldsymbol{J}$ depend on the thickness coordinate $z$ and on the time $t$,

$$
R=R(z, t), \quad J=J(z, t) .
$$

In addition, a density function must be considered. Let us assume the simplest case - the density depends only on the thickness coordinate

$$
\rho_{0}=\rho_{0}(z)
$$

$\rho_{0}$ is the density of the three-dimensional solid.

Using the Laplace transform of a function $f(t)$,

$$
\bar{f}(s)=\int_{0}^{\infty} f(t) e^{-s t} \mathrm{~d} t
$$

one can write Eqs. (15), (16) in the form $[12,18]$

$$
\overline{\boldsymbol{\sigma}}=s \overline{\boldsymbol{R}}(s) \cdot \cdot \bar{\varepsilon}, \quad \overline{\boldsymbol{\varepsilon}}=s \overline{\boldsymbol{J}}(s) \cdot \cdot \bar{\sigma} .
$$

Let us consider an orthotropic viscoelastic material. Using the correspondence principle we may write down the constitutive equations for the Laplace mappings in the following form:

$$
\begin{aligned}
& \bar{\varepsilon}_{1}=\frac{1}{s \bar{E}_{1}} \bar{\sigma}_{1}-\frac{\bar{\nu}_{21}}{s \bar{E}_{2}} \bar{\sigma}_{2}-\frac{\bar{\nu}_{n 1}}{s \bar{E}_{n}} \bar{\sigma}_{n}, \\
& \bar{\varepsilon}_{2}=\frac{1}{s \bar{E}_{2}} \bar{\sigma}_{2}-\frac{\bar{\nu}_{12}}{s \bar{E}_{1}} \bar{\sigma}_{1}-\frac{\bar{\nu}_{n 2}}{s \bar{E}_{n}} \bar{\sigma}_{n}, \\
& \bar{\varepsilon}_{n}=\frac{1}{s \bar{E}_{n}} \bar{\sigma}_{n}-\frac{\bar{v}_{1 n}}{s \bar{E}_{1}} \bar{\sigma}_{1}-\frac{\bar{v}_{2 n}}{s \bar{E}_{2}} \bar{\sigma}_{2}, \\
& \bar{\varepsilon}_{12}=\frac{\bar{\sigma}_{12}}{s \bar{G}_{12}}, \\
& \bar{\varepsilon}_{n 1}=\frac{\bar{\sigma}_{n 1}}{s \bar{G}_{n 1}}, \\
& \bar{\varepsilon}_{2 n}=\frac{\bar{\sigma}_{2 n}}{s \bar{G}_{2 n}}
\end{aligned}
$$

with $\bar{\nu}_{i j} \bar{E}_{j}=\bar{v}_{j i} \bar{E}_{i}$. 
Using the analogy between (19) or (20) and Hooke's law we can extend the identification procedure $[5,6,38]$ to the Laplace mapping of the effective relaxation or creep functions, see [2]. The in-plane and the out-of-plane stiffness tensor components are

$$
\begin{array}{ll}
\bar{A}_{11}=\frac{1}{4}\left\langle\frac{\bar{E}_{1}+\bar{E}_{2}+2 \bar{E}_{1} \bar{v}_{21}}{1-\bar{v}_{12} \bar{v}_{21}}\right\rangle, & \bar{A}_{12}=\frac{1}{4}\left\langle\frac{\bar{E}_{1}-\bar{E}_{2}}{1-\bar{v}_{12} \bar{v}_{21}}\right\rangle, \\
\bar{A}_{22}=\frac{1}{4}\left\langle\frac{\bar{E}_{1}+\bar{E}_{2}-2 \bar{E}_{1} \bar{v}_{21}}{1-\bar{v}_{12} \bar{v}_{21}}\right\rangle, & \bar{A}_{44}=\left\langle\bar{G}_{12}\right\rangle, \\
\bar{B}_{13}=-\frac{1}{4}\left\langle\frac{\bar{E}_{1}+\bar{E}_{2}+2 \bar{E}_{1} \bar{v}_{21}}{1-\bar{v}_{12} \bar{v}_{21}} z\right\rangle, & -\bar{B}_{23}=\bar{B}_{14}=\frac{1}{4}\left\langle\frac{\bar{E}_{1}-\bar{E}_{2}}{1-\bar{v}_{12} \bar{v}_{21}} z\right\rangle, \\
\bar{B}_{24}=\frac{1}{4}\left\langle\frac{\bar{E}_{1}+\bar{E}_{2}-2 \bar{E}_{1} \bar{v}_{21}}{1-\bar{v}_{12} \bar{v}_{21}} z\right\rangle, & \bar{B}_{42}=-\left\langle\bar{G}_{12} z\right\rangle, \\
\bar{C}_{33}=\frac{1}{4}\left\langle\frac{\bar{E}_{1}+\bar{E}_{2}+2 \bar{E}_{1} \bar{v}_{21}}{1-\bar{v}_{12} \bar{v}_{21}} z^{2}\right\rangle, & \bar{C}_{34}=-\frac{1}{4}\left\langle\frac{\bar{E}_{1}-\bar{E}_{2}}{1-\bar{v}_{12} \bar{v}_{21}} z^{2}\right\rangle, \\
\bar{C}_{44}=\frac{1}{4}\left\langle\frac{\bar{E}_{1}+\bar{E}_{2}-2 \bar{E}_{1} \bar{v}_{21}}{1-\bar{v}_{12} \bar{\nu}_{21}} z^{2}\right\rangle, & \bar{C}_{22}=\left\langle\bar{G}_{12} z^{2}\right\rangle,
\end{array}
$$

while the transverse shear stiffness tensor components are

$$
\bar{\Gamma}_{1}=\frac{1}{2}\left(\lambda^{2}+\eta^{2}\right) \frac{\bar{A}_{44} \bar{C}_{22}-\bar{B}_{42}^{2}}{\bar{A}_{44}}, \quad \bar{\Gamma}_{2}=\frac{1}{2}\left(\eta^{2}-\lambda^{2}\right) \frac{\bar{A}_{44} \bar{C}_{22}-\bar{B}_{42}^{2}}{\bar{A}_{44}}
$$

where $\lambda$ and $\eta$ are the minimal non-zero eigen-values following from the Sturm-Liouville problems

$$
\begin{aligned}
& \frac{\mathrm{d}}{\mathrm{d} z}\left(\bar{G}_{2 n} \frac{\mathrm{d} Z}{\mathrm{~d} z}\right)+\lambda^{2} \bar{G}_{12} Z=0,\left.\quad \frac{\mathrm{d} Z}{\mathrm{~d} z}\right|_{|z|=\frac{h}{2}}=0, \\
& \frac{\mathrm{d}}{\mathrm{d} z}\left(\bar{G}_{1 n} \frac{\mathrm{d} \tilde{Z}}{\mathrm{~d} z}\right)+\eta^{2} \bar{G}_{12} \tilde{Z}=0,\left.\quad \frac{\mathrm{d} \tilde{Z}}{\mathrm{~d} z}\right|_{|z|=\frac{h}{2}}=0 .
\end{aligned}
$$

Here $\langle(\ldots)\rangle=\int_{-h / 2}^{h / 2}(\ldots) \mathrm{d} z, h$ is the thickness of the plate.

In the case of isotropic material behavior one has to set in Eqs. (20)

$$
\bar{E}_{1}=\bar{E}_{2}=\bar{E}_{n}=\bar{E}(z, s), \quad \bar{v}_{i j}=\bar{v}(z, s), \quad \bar{G}_{12}=\bar{G}_{n 1}=\bar{G}_{2 n}=\bar{\mu}(z, s)=\frac{\bar{E}(z, s)}{2[1+\bar{v}(z, s)]} .
$$

Instead of (21), (22) the following non-zero components of the stiffness tensors are valid:

- the in-plane stiffness tensor components

$$
\bar{A}_{11}=\frac{1}{2}\left\langle\frac{\bar{E}}{1-\bar{v}}\right\rangle, \quad \bar{A}_{22}=\frac{1}{2}\left\langle\frac{\bar{E}}{1+\bar{v}}\right\rangle=\bar{A}_{44}=\langle\bar{\mu}\rangle,
$$

- the coupling stiffness tensor components

$$
\bar{B}_{13}=-\frac{1}{2}\left\langle\frac{\bar{E}}{1-\bar{v}} z\right\rangle, \quad \bar{B}_{24}=\frac{1}{2}\left\langle\frac{\bar{E}}{1+\bar{v}} z\right\rangle=-\bar{B}_{42}=\langle\bar{\mu} z\rangle
$$


- the plate stiffness tensor components

$$
\bar{C}_{33}=\frac{1}{2}\left\langle\frac{\bar{E}}{1-\bar{v}} z^{2}\right\rangle, \quad \bar{C}_{44}=\frac{1}{2}\left\langle\frac{\bar{E}}{1+\bar{v}} z^{2}\right\rangle=\bar{C}_{22}=\left\langle\bar{\mu} z^{2}\right\rangle,
$$

- the transverse shear stiffness tensor components

$$
\bar{\Gamma}_{1}=\bar{\Gamma}=\lambda^{2} \frac{\bar{A}_{44} \bar{C}_{22}-\bar{B}_{42}^{2}}{\bar{A}_{44}}
$$

with $\lambda$ following from

$$
\frac{\mathrm{d}}{\mathrm{d} z}\left(\bar{\mu} \frac{\mathrm{d} Z}{\mathrm{~d} z}\right)+\lambda^{2} \bar{\mu} Z=0,\left.\quad \frac{\mathrm{d} Z}{\mathrm{~d} z}\right|_{|z|=\frac{h}{2}}=0 .
$$

For the plate which is symmetrically to the midplane the relation $B=0$ holds true. The relaxation functions of the isotropic viscoelastic plate with symmetric cross-section were considered in [8].

The tensors of inertia and the plate density are given by Altenbach and Zhilin [1], Altenbach and Eremeyev [7], Zhilin [38]

$$
\rho=\left\langle\rho_{0}\right\rangle, \quad \rho \Theta_{1}=-\left\langle\rho_{0} z\right\rangle \boldsymbol{c}, \quad \rho \Theta_{2}=\Theta \boldsymbol{a}, \quad \Theta=\left\langle\rho_{0} z^{2}\right\rangle .
$$

Considering the symmetry of the thickness geometry and of the material properties of the plate from (29) one gets that $\Theta_{1}=\mathbf{0}$. $\Theta$ characterizes the rotatory inertia of the cross-section of the plate.

Note that for isotropic viscoelastic material we introduced three functions $\bar{E}(s), \bar{\mu}(s)$ and $\bar{v}(s)$. They are interlinked by the formula

$$
\bar{E}=2 \bar{\mu}(1+\bar{v})
$$

Following [21,22] we use Eq. (30) as the definition of the Poisson's ratio for viscoelastic material.

In the theory of viscoelasticity of solids the assumption $v(t)=v=$ const is often used. It is fulfilled in many applications (see arguments in $[12,15,37]$ concerning $v(t) \approx$ const). For example, $v=1 / 2$ for an incompressible viscoelastic material. In the general case, $v$ is a function of $t . v(t)$ was considered as an increasing function of $t[11,32,37]$ or non-monotonous function of $t[21,22]$. The latter case may be realized for cellular materials or foams. Further we consider the influence of $v(t)$ on the deflexion of the viscoelastic plate and its effective relaxation functions.

\section{Bounds for the eigen-values}

To obtain the dependence of the transverse shear stiffness relaxation function we have to solve Eq. (28). In the general case, the solution of the spectral problem (28) may be performed numerically. For example, in [7] the shooting method [35] was used. Let us note that for the viscoelastic plate $\bar{\mu}=\bar{\mu}(z, s)$. Thus, $\lambda=\lambda(s)$. It means that for the determination of $\Gamma(t)$ one has to solve (28) for any arbitrary value of $s$ and with the help of $\lambda=\lambda(s)$ to find numerically the inverse Laplace transform of $\bar{\Gamma}$.

Let us find the bounds for the values of $\lambda$. Introducing a new independent variable $\zeta$ by the formula

$$
\zeta=\int_{-h / 2}^{z} \frac{\mathrm{d} z}{\bar{\mu}(z, s)}
$$

one can transform (28) to the form (see, for example, [17] for details)

$$
\frac{\mathrm{d}^{2} Z}{\mathrm{~d} \zeta^{2}}+\lambda^{2} \bar{\mu}(z, s)^{2} Z=0,\left.\quad \frac{\mathrm{d} Z}{\mathrm{~d} \zeta}\right|_{\zeta=0, L}=0
$$


Here

$$
L=L(s) \equiv \int_{-h / 2}^{h / 2} \frac{\mathrm{d} z}{\bar{\mu}(z, s)}
$$

Substituting $\zeta=\zeta / L$, one can transform the spectral problem (31) to the canonical form

$$
\frac{\mathrm{d}^{2} Z}{\mathrm{~d} \zeta^{2}}+\lambda^{2} L(s)^{2} \bar{\mu}(z, s)^{2} Z=0,\left.\quad \frac{\mathrm{d} Z}{\mathrm{~d} \zeta}\right|_{\zeta=0,1}=0 .
$$

The following theorem exists [13]:

Theorem If one has two eigen-value problems

$$
\frac{\mathrm{d}^{2} Z}{\mathrm{~d} \zeta^{2}}+\lambda^{2} f_{1} Z=0, \quad \frac{\mathrm{d}^{2} Z}{\mathrm{~d} \zeta^{2}}+\lambda^{2} f_{2} Z=0,\left.\quad \frac{\mathrm{d} Z}{\mathrm{~d} \zeta}\right|_{\zeta=0,1}=0
$$

with two functions $f_{1}(\zeta)$ and $f_{2}(\zeta)$ such that $f_{1} \leq f_{2}$, then the following inequality holds true $\lambda_{1} \geq \lambda_{2}$. Here $\lambda_{1}$ and $\lambda_{2}$ are the eigen-values corresponding to the functions $f_{1}(\zeta)$ and $f_{2}(\zeta)$, respectively.

Applying this theorem to Eq. (32) and using the inequalities $\mu_{\min }(s) \leq \bar{\mu}(z, s) \leq \mu_{\max }(s)$, we obtain the lower and upper bounds of $\lambda$

$$
\frac{\pi}{L(s) \mu_{\max }(s)} \leq \lambda(s) \leq \frac{\pi}{L(s) \mu_{\min }(s)}
$$

For a homogeneous plate $\mu_{\min }=\mu_{\max }=\bar{\mu}(s), L(s)=h / \mu(s)$, and both bounds coincide with each other.

\section{Quasi-static behavior of a symmetric orthotropic plate}

Let us consider the quasi-static deformations of a symmetric orthotropic plate. In this case Eqs. $(7.1,2)$ split into two parts: the in-plane problem for tangential displacements $u_{1}$ and $u_{2}$, and the bending problem for $w$, $\varphi_{1}$ and $\varphi_{2}$, respectively.

The constitutive equations for a symmetric orthotropic plate can be given as follows:

$$
\begin{aligned}
& \bar{T}_{1}=s\left(\bar{A}_{11}+2 \bar{A}_{12}+\bar{A}_{22}\right) \bar{\mu}_{1}+s\left(\bar{A}_{11}-\bar{A}_{22}\right) \bar{\mu}_{2}, \\
& \bar{T}_{2}=s\left(\bar{A}_{11}-\bar{A}_{22}\right) \bar{\mu}_{1}+s\left(\bar{A}_{11}-2 \bar{A}_{12}+\bar{A}_{22}\right) \bar{\mu}_{2}, \\
& \bar{T}_{12}=2 s \bar{A}_{44} \bar{\mu}_{12}, \\
& \bar{T}_{1 n}=s\left(\bar{\Gamma}_{1}+\bar{\Gamma}_{2}\right) \bar{\gamma}_{1}, \bar{T}_{2 n}=s\left(\bar{\Gamma}_{1}-\bar{\Gamma}_{2}\right) \bar{\gamma}_{2}, \\
& \bar{M}_{1}=s\left(\bar{C}_{33}-2 \bar{C}_{34}+\bar{C}_{44}\right) \bar{\kappa}_{1}+s\left(\bar{C}_{33}-\bar{C}_{44}\right) \bar{\kappa}_{2}, \\
& \bar{M}_{2}=s\left(\bar{C}_{33}-\bar{C}_{44}\right) \bar{\kappa}_{1}+s\left(\bar{C}_{33}+2 \bar{C}_{34}+\bar{C}_{44}\right) \bar{\kappa}_{2}, \\
& \bar{M}_{12}=s \bar{C}_{22}\left(\bar{\kappa}_{12}+\bar{\kappa}_{21}\right) .
\end{aligned}
$$

In Cartesian coordinates with the geometrical relations (11) Eqs. $(7.1,2)$ reduce to the form

$$
\begin{aligned}
& s\left(\bar{A}_{11}+2 \bar{A}_{12}+\bar{A}_{22}\right) \bar{u}_{1,11}+s\left(\bar{A}_{11}-A_{22}\right) \bar{u}_{2,21}+s \bar{A}_{44}\left(\bar{u}_{1,21}+\bar{u}_{2,11}\right)+\bar{q}_{1}=0, \\
& s\left(\bar{A}_{11}-\bar{A}_{22}\right) \bar{u}_{1,12}+s\left(\bar{A}_{11}-2 \bar{A}_{12}+\bar{A}_{22}\right) u_{2,22}+s \bar{A}_{44}\left(\bar{u}_{1,22}+\bar{u}_{2,12}\right)+\bar{q}_{2}=0 .
\end{aligned}
$$

Equation (7.3) has the following form:

$$
\left(\bar{\Gamma}_{1}+\bar{\Gamma}_{2}\right) \bar{w}_{, 11}+\left(\bar{\Gamma}_{1}-\bar{\Gamma}_{2}\right) \bar{w}_{, 22}+\left(\bar{\Gamma}_{1}+\bar{\Gamma}_{2}\right) \bar{\varphi}_{1,1}+\left(\bar{\Gamma}_{1}-\bar{\Gamma}_{2}\right) \bar{\varphi}_{2,2}+\bar{q}_{n} / s=0 .
$$


Equations $(7.4,5)$ result in

$$
\begin{gathered}
s\left(\bar{C}_{33}-2 \bar{C}_{34}+\bar{C}_{44}\right) \bar{\varphi}_{1,11}+s\left(\bar{C}_{33}-\bar{C}_{44}\right) \bar{\varphi}_{2,21} \\
+s \bar{C}_{22}\left(\bar{\varphi}_{1,22}+\bar{\varphi}_{2,12}\right)-s\left(\bar{\Gamma}_{1}+\bar{\Gamma}_{2}\right) \bar{\varphi}_{1}-s\left(\bar{\Gamma}_{1}+\bar{\Gamma}_{2}\right) \bar{w}_{11}+\bar{m}_{1}=0, \\
s\left(\bar{C}_{33}+2 \bar{C}_{34}+\bar{C}_{44}\right) \bar{\varphi}_{2,22}+s\left(\bar{C}_{33}-\bar{C}_{44}\right) \bar{\varphi}_{1,12} \\
+s \bar{C}_{22}\left(\bar{\varphi}_{1,21}+\bar{\varphi}_{2,11}\right)-s\left(\bar{\Gamma}_{1}-\bar{\Gamma}_{2}\right) \bar{\varphi}_{2}-s\left(\bar{\Gamma}_{1}-\bar{\Gamma}_{2}\right) \bar{w}_{, 2}+\bar{m}_{2}=0 .
\end{gathered}
$$

Let us eliminate the functions $\bar{\varphi}_{1}$ and $\bar{\varphi}_{2}$ from Eqs. (37) and (38). For brevity, let us assume that $\bar{m}_{1}=0$, $\bar{m}_{2}=0$. By using operator notations, Eqs. (38) may be rewritten in the form

$$
L_{11} \bar{\varphi}_{1}+L_{12} \bar{\varphi}_{2}=b_{1} \bar{w}, \quad L_{21} \bar{\varphi}_{1}+L_{22} \bar{\varphi}_{2}=b_{2} \bar{w}
$$

where

$$
\begin{aligned}
L_{11} & =s s\left(\bar{C}_{33}-2 \bar{C}_{34}+\bar{C}_{44}\right) \partial_{1}^{2}+s \bar{C}_{22} \partial_{2}^{2}-s \bar{\Gamma}_{1}-s \bar{\Gamma}_{2}, \\
L_{22} & =s\left(\bar{C}_{33}+2 \bar{C}_{34}+\bar{C}_{44}\right) \partial_{2}^{2}+s \bar{C}_{22} \partial_{1}^{2}-s \bar{\Gamma}_{1}+s \bar{\Gamma}_{2}, \\
L_{21} & =L_{12}=\left(C_{33}+C_{22}-C_{44}\right) \partial_{1} \partial_{2}, \\
b_{1} & =s\left(\bar{\Gamma}_{1}+\bar{\Gamma}_{2}\right) \partial_{1}, \quad b_{2}=s\left(\bar{\Gamma}_{1}-\bar{\Gamma}_{2}\right) \partial_{2}, \\
\partial_{\alpha}(\ldots) & \equiv(\ldots)_{, \alpha}, \quad \alpha=1,2 .
\end{aligned}
$$

From (39) we obtain the relations $\mathcal{L} \bar{\varphi}_{1}=\mathcal{L}_{1} \bar{w}, \mathcal{L} \bar{\varphi}_{2}=\mathcal{L}_{2} \bar{w}$, where

$$
\mathcal{L}=L_{11} L_{22}-L_{12}^{2}, \quad \mathcal{L}_{1}=L_{22} b_{1}-L_{12} b_{2}, \quad \mathcal{L}_{2}=L_{11} b_{2}-L_{21} b_{1}
$$

Using operator notations Eq. (37) can be rewritten as follows:

$$
\mathcal{L}_{w} \bar{w}+b_{1} \bar{\varphi}_{1}+b_{2} \bar{\varphi}_{2}+\bar{q}_{n}=0
$$

where $\mathcal{L}_{w}=s\left(\bar{\Gamma}_{1}+\bar{\Gamma}_{2}\right) \partial_{1}^{2}+s\left(\bar{\Gamma}_{1}-\bar{\Gamma}_{2}\right) \partial_{2}^{2}$. Then we obtain one differential equation of sixth order with respect to $\bar{w}$,

$$
\left(\mathcal{L} \mathcal{L}_{w}+b_{1} \mathcal{L}_{1}+b_{2} \mathcal{L}_{2}\right) \bar{w}+\mathcal{L} \bar{q}_{n}=0 .
$$

For the isotropic plate we have $\bar{C}_{22}=\bar{C}_{44}, \bar{C}_{34}=0, \bar{\Gamma}_{2}=0$, and one gets

$$
\begin{array}{ll}
L_{11}=\left(s \bar{C}_{33}+\bar{C}_{44}\right) \partial_{1}^{2}+s \bar{C}_{44} \partial_{2}^{2}-s \bar{\Gamma}_{1}, & L_{22}=s\left(\bar{C}_{33}+\bar{C}_{44}\right) \partial_{2}^{2}+s \bar{C}_{44} \partial_{1}^{2}-s \bar{\Gamma}_{1}, \\
L_{21}=L_{12}=C_{33} \partial_{1} \partial_{2}, & b_{2}=s \bar{\Gamma}_{1} \partial_{2}, \\
b_{1}=s \bar{\Gamma}_{1} \partial_{1}, &
\end{array}
$$

and

$$
\begin{aligned}
& \mathcal{L}=s^{2}\left[\left(\bar{C}_{33}+\bar{C}_{44}\right) \partial_{1}^{2}+\bar{C}_{44} \partial_{2}^{2}-\bar{\Gamma}_{1}\right]\left[\left(\bar{C}_{33}+\bar{C}_{44}\right) \partial_{2}^{2}+\bar{C}_{44} \partial_{1}^{2}-\bar{\Gamma}_{1}\right]-s^{2} \bar{C}_{33}^{2} \partial_{1}^{2} \partial_{2}^{2}, \\
& \mathcal{L}_{1}=s^{2} \bar{\Gamma}_{1}\left[\left(\bar{C}_{33}+\bar{C}_{44}\right) \partial_{2}^{2}+\bar{C}_{44} \partial_{1}^{2}-\bar{\Gamma}_{1}\right] \partial_{1}+s^{2} \bar{\Gamma}_{1} \bar{C}_{33} \partial_{1} \partial_{2}^{2}, \\
& \mathcal{L}_{2}=-s^{2} \bar{\Gamma}_{1} \bar{C}_{33} \partial_{1}^{2} \partial_{2}+s^{2} \bar{\Gamma}_{1}\left[\left(\bar{C}_{33}+\bar{C}_{44}\right) \partial_{1}^{2}+\bar{C}_{44} \partial_{2}^{2}-\bar{\Gamma}_{1}\right] \partial_{2} .
\end{aligned}
$$

Finally, the bending Eq. (40) has the form

$$
s\left(\bar{C}_{33}+\bar{C}_{44}\right) \Delta \Delta \bar{w}=\partial_{1} \bar{m}_{1}+\partial_{2} \bar{m}_{2}-\frac{\bar{C}_{33}+\bar{C}_{44}}{\bar{\Gamma}_{1}} \Delta \bar{q}_{n}+\bar{q}_{n} .
$$

If $m_{1}=m_{2}=0$ and $\bar{\Gamma}_{1} \rightarrow \infty$ one gets the Kirchhoff's plate equation

$$
s \bar{D} \Delta \Delta \bar{w}=\bar{q}_{n}
$$

with the bending stiffness $\bar{D}=\bar{C}_{33}+\bar{C}_{44}$. 


\section{Examples of effective stiffness relaxation functions}

\subsection{Homogeneous plate}

The simplest test for the correctness of the estimated stiffness properties is the homogeneous isotropic plate. The basic geometrical property is the thickness $h$. The plate is symmetrically with respect to the mid-plane. All material properties are constant over the thickness, that means they do not depend on the thickness coordinate.

For the sake of simplicity, at first let us consider the case $v(t)=v=$ const. That means that the following relations hold true: $E(t)=2 \mu(t)(1+v)$. The non-zero components of the classical relaxation tensors are

$$
\begin{aligned}
& A_{11}(t)=\frac{E(t) h}{2(1-v)}, \quad A_{22}(t)=\frac{E(t) h}{2(1+v)}=\mu(t) h, \\
& C_{33}(t)=\frac{E(t) h^{3}}{24(1-v)}, \quad C_{22}(t)=\frac{E(t) h^{3}}{24(1+v)}=\frac{\mu(t) h^{3}}{12} .
\end{aligned}
$$

Thus, the bending stiffness results in

$$
D(t)=\frac{E(t) h^{3}}{12\left(1-v^{2}\right)} .
$$

The density and the rotatory inertia coefficient are

$$
\rho=\rho_{0} h, \quad \Theta=\frac{\rho_{0} h^{3}}{12} .
$$

The transverse shear relaxation function follows from (27). The solution of (28) with $\bar{\mu}=\bar{\mu}(s)$ is given by $\cos \lambda z=0$. It yields the smallest eigenvalue $\lambda=\pi / h$ which does not depend on $s$. Finally, one obtains

$$
\Gamma(t)=\lambda^{2} C_{22}=\frac{\pi^{2}}{h^{2}} \frac{\mu(t) h^{3}}{12}=\frac{\pi^{2}}{12} \mu(t) h .
$$

$\pi^{2} / 12$ is a factor which is similar to the shear correction factor which was first introduced by Timoshenko [36] in the theory of beams. Here this factor is a result of the non-classical establishments of the transverse shear stiffness. Comparing this value with Mindlin's estimate $\pi^{2} / 12$ [26] and Reissner's estimate 5/6 [29,30] one concludes that the direct approach yields the same value like in Mindlin's theory (note that Mindlin's shear correction is based on the solution of a dynamic problem, here the solution of a static problem was used). The Reissner's value slightly differs.

It is evident that in the case of homogeneous viscoelastic plates with constant Poisson's ratio one gets the same relations for the effective stiffness tensors as in the case of elastic plates $[5,6]$. There is only one difference-they are now functions of $t$.

At second, let us consider the general case $v=v(t)$. In this case $D$ is reconstructed from

$$
\bar{D}=\frac{\bar{E} h^{3}}{12\left(1-\bar{v}^{2}\right)}
$$

as follows:

$$
D(t)=\int_{-\infty}^{t} \frac{E(t-\tau) h^{3}}{12\left[1-v^{2}(\tau)\right]} \mathrm{d} \tau
$$

Using the initial value theorem that $f(0)=\lim _{s \rightarrow \infty} s \bar{f}(s)$ and the final value theorem that $\lim _{t \rightarrow \infty} f(t)=$ $\lim _{s \rightarrow o} s \bar{f}(s)$ we establish that

$$
D(0)=\frac{E(0) h^{3}}{12\left(1-v_{0}^{2}\right)}, \quad D(\infty)=\frac{E(\infty) h^{3}}{12\left(1-v_{\infty}^{2}\right)},
$$


where

$$
v_{0}=\frac{E(0)}{2 \mu(0)}-1, \quad v_{\infty}=\frac{E(\infty)}{2 \mu(\infty)}-1
$$

$v_{\infty}$ may be considered as Poisson's ratio in the relaxed state, while $v_{0}$ is Poisson's ration in the initial state. For the sake of simplicity we use the notation $f(\infty)=\lim _{t \rightarrow \infty} f(t)$.

As an example let us consider the relaxation functions following from the standard linear viscoelastic model $[15,18,28,37]$

$$
E(t)=E_{\infty}+\left(E_{0}-E_{\infty}\right) e^{-t / \tau_{\mathrm{E}}}, \quad \mu(t)=\mu_{\infty}+\left(\mu_{0}-\mu_{\infty}\right) e^{-t / \tau_{\mu}},
$$

where $E_{\infty}$ and $E_{0}$ are the equilibrium and the short-time Young's moduli ( $E_{\infty}<E_{0}$ ), while $\mu_{\infty}$ and $\mu_{0}$ are the equilibrium and the short-time shear moduli $\left(\mu_{\infty}<\mu_{0}\right)$, respectively. $\tau_{\mathrm{E}}$ and $\tau_{\mu}$ are the relaxation times for tension and shear. Using the Laplace transforms

$$
\bar{E}=\frac{E_{\infty}}{s}+\frac{E_{0}-E_{\infty}}{s+\tau_{\mathrm{E}}}, \quad \bar{\mu}=\frac{\mu_{\infty}}{s}+\frac{\mu_{0}-\mu_{\infty}}{s+\tau_{\mu}}
$$

we obtain that

$$
\bar{v}=\frac{\left(s+\tau_{\mu}\right)\left(E_{\infty} \tau_{\mathrm{E}}+E_{0} s\right)}{2\left(s+\tau_{\mathrm{E}}\right)\left(\mu_{\infty} \tau_{\mu}+\mu_{0} s\right)}-1 .
$$

Note that the assumption $\bar{v}=$ const is fulfilled if and only if $E_{\infty} / E_{0}=\mu_{\infty} / \mu_{0}$ and $\tau_{\mathrm{E}}=\tau_{\mu}$. Finally, we obtain the expression for the Laplace transform of the bending stiffness,

$$
\bar{D}=\frac{\bar{\mu}^{2} h^{3}}{3(4 \bar{\mu}-\bar{E})}=\frac{\left(\mu_{\infty}+\mu_{0} \tau_{\mu} s\right)^{2} h^{3}}{s^{2}\left(s \tau_{\mu}+1\right)^{2}\left[12 \frac{\mu_{\infty}+\mu_{0} \tau_{\mu} s}{s\left(s \tau_{\mu}+1\right)}-3 \frac{E_{\infty}+E_{0} \tau_{\mathrm{E}} s}{s\left(s \tau_{\mathrm{E}}+1\right)}\right]}
$$

To avoid an awkward expression we do not present the expression for $D(t)$ corresponding to the latter equation. Here we have the following relations:

$$
D(0)=\frac{E_{0} h^{3}}{12\left(1-v_{0}^{2}\right)}, \quad D(\infty)=\frac{E_{\infty} h^{3}}{12\left(1-v_{\infty}^{2}\right)},
$$

where

$$
v_{0}=\frac{E_{0}}{2 \mu_{0}}-1, \quad v_{\infty}=\frac{E_{\infty}}{2 \mu_{\infty}}-1 .
$$

The dependence of the dimensionless bending stiffness on time is presented in Fig. 3 (solid line). Here the following values are assumed $v_{0}=0.2, v_{\infty}=0.4, \mu_{\infty}=\mu_{0} / 2, \tau_{\mu}=\tau_{\mathrm{E}}$. We also present the two curves of $D(t)$ in the case of constant Poisson's ratios which are equal to 0.1, 0.4 (Fig. 3, dashed lines). Note that in the case $v \neq$ const $D(t)$ is a non-monotonous function of $t$, while $D(t)$ is the monotonous decreasing function for constant Poisson's ratio.

\subsection{Functionally graded material}

In this paragraph we consider small deformations of an FGM plate made of a viscoelastic polymer foam. For the panel made from a porous polymer foam the distribution of the pores over the thickness can be inhomogeneous (see, for example, Fig. 2). Let us introduce $h$ as the thickness of the panel, $\rho_{\mathrm{s}}$ as the density of the bulk material and $\rho_{\mathrm{p}}$ as the minimum value of the density of the foam. For the description of the symmetric distribution of the porosity we assume the power law [7]

$$
V(z)=\alpha+(1-\alpha)\left|\frac{2 z}{h}\right|^{n},
$$




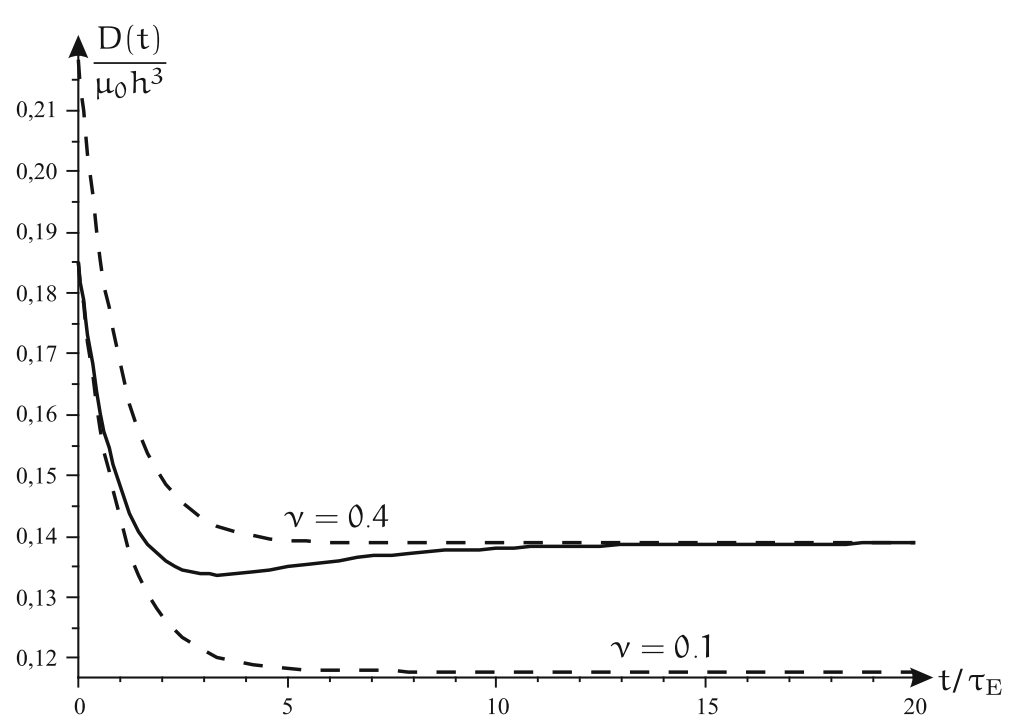

Fig. 3 Dimensionless bending stiffness in dependence on time for constant Poisson's ratio (dashed lines) and in the general case (solid line)

where $\alpha=\rho_{\mathrm{p}} / \rho_{\mathrm{s}}$ is the minimal relative density, $n$ is the power. $n=0$ corresponds to the homogeneous plate described in the previous paragraph.

The properties of the foam strongly depend on the porosity and the cell structure. For the polymer foam in [16] the modification of the standard linear viscoelastic solid is proposed. For the open-cell foam the constitutive law has the form

$$
\dot{\sigma}+\tau_{\mathrm{E}} \sigma=C_{1} V(z)^{2}\left[E_{\infty} \tau_{\mathrm{E}} \varepsilon+E_{0} \dot{\varepsilon}\right],
$$

while for the closed-cell foam the constitutive equation has the form

$$
\dot{\sigma}+\tau_{\mathrm{E}} \sigma=C_{2}\left[\phi^{2} V(z)^{2}+(1-\phi) V(z)\right]\left[E_{\infty} \tau_{\mathrm{E}} \varepsilon+E_{0} \dot{\varepsilon}\right] .
$$

Here $C_{1} \approx 1, C_{2} \approx 1, \phi$ describes the relative volume of the solid polymer concentrated near the cell ribs. Usually, $\phi=0.6 \ldots 0.7 . E_{\infty}, E_{0}, \tau_{\mathrm{E}}$ are material constants of the polymer used in manufacturing of the foam.

From Eqs. (46), (47) one can see that the corresponding relaxation function is given by the relations

$$
E=E(z, t)=E(t) \kappa(z)
$$

where $E(t)$ is defined by Eq. (44), while

$$
\kappa(z)=C_{1} V(z)^{2}
$$

for open-cell foam and

$$
\kappa(z)=C_{2}\left[\phi^{2} V(z)^{2}+(1-\phi) V(z)\right]
$$

for closed-cell foam, respectively. Analogous to (48) the following relation can be established for the shear relaxation function:

$$
\mu=\mu(z, t)=\mu(t) m(z)
$$

Equations (48) and (49) have the meaning that the viscoelastic properties of the foam, for example, the time of relaxation, do not depend on the porosity distribution. Note that representations (48) and (49) are only simple assumptions for spatial non-homogeneous foams.

Using experimental data presented in $[9,16]$ one can assume $v=$ const. In this case we obtain that $A_{11}$, $A_{22}, C_{33}, C_{22}$ are related to

$$
A_{11}=\frac{1+v}{1-v} A_{22}, \quad C_{33}=\frac{1+v}{1-v} C_{22},
$$


For the open-cell foam $A_{22}$ and $C_{22}$ are given by

$$
A_{22}=h\left[\alpha^{2}+\frac{2 \alpha(1-\alpha)}{n+1}+\frac{(1-\alpha)^{2}}{2 n+1}\right] \mu(t), \quad C_{22}=\frac{h^{3}}{12}\left[\alpha^{2}+\frac{6 \alpha(1-\alpha)}{n+3}+\frac{3(1-\alpha)^{2}}{2 n+3}\right] \mu(t),
$$

while for the closed-cell foam by

$$
\begin{aligned}
& A_{22}=h\left\{\phi^{2}\left[\alpha^{2}+\frac{2 \alpha(1-\alpha)}{n+1}+\frac{(1-\alpha)^{2}}{2 n+1}\right]+(1-\phi)\left[\alpha+\frac{1-\alpha}{n+1}\right]\right\} \mu(t), \\
& C_{22}=\frac{h^{3}}{12}\left\{\phi^{2}\left[\alpha^{2}+\frac{6 \alpha(1-\alpha)}{n+3}+\frac{3(1-\alpha)^{2}}{2 n+3}\right]+(1-\phi)\left[\alpha+\frac{3(1-\alpha)}{n+3}\right]\right\} \mu(t) .
\end{aligned}
$$

Here we assume that $C_{1}=1, C_{2}=1$, and that $\phi$ does not depend on $z$.

From Eqs. (51), (52) it is easy to see that the classical relaxation functions differ only by factors from the shear relaxation function. Note that one can easily extend Eqs. (46), (47) to the case of general constitutive Eqs. (15) or (18). Thus, using the assumption that $v=$ const, one can calculate the classical effective stiffness relaxation functions for general viscoelastic constitutive equations multiplying the shear relaxation function with the corresponding factor similar to Eqs. (51), (52). In the more general situation with $v=v(t)$ or taking into account other viscoelastic phenomena, for example, the filtration of a fluid in the saturated foam, the effective stiffness relaxation functions may be more complex than for the pure solid polymer discussed here.

Finally, we should mention that in the case of constant Poisson's ratio and with the assumption (49) the determination of the effective in-plane, bending and transverse shear stiffness tensors of a symmetric FGM viscoelastic plate made of a polymer foam can be realized by the same method as for elastic plates [5-7]. The relaxation functions for viscoelastic FGM plates can be found from the values of the corresponding effective stiffness of an elastic FGM plate by multiplication with the normalized shear relaxation function of the polymer solid.

\section{Bending of viscoelastic plate}

\subsection{Homogeneous plate}

Let us assume the plate bending with $m=0$. From Eq. (41) we obtain that

$$
s \bar{D} \Delta \Delta \bar{w}=\bar{q}_{n}-\frac{\bar{D}}{\bar{\Gamma}_{1}} \Delta \bar{q}_{n}
$$

where $\bar{D}=\bar{E} h^{3} / 12\left(1-\bar{v}^{2}\right)$ is Laplace transform of the bending stiffness relaxation function, $\bar{\Gamma}_{1}=\pi^{2} \bar{\mu} h / 12$ is Laplace transform of the shear stiffness relaxation function, $\bar{w}=\bar{u} \cdot n$ is the Laplace transform of the plate deflection, $\bar{q}_{n}=\overline{\boldsymbol{q}} \cdot \boldsymbol{n}$ is Laplace transform of the transverse load, respectively. Using Eqs. (30), (53) transforms to

$$
s \bar{D} \Delta \Delta \bar{w}=\bar{q}_{n}-\frac{2 h^{2}}{\pi^{2}(1-\bar{v})} \Delta \bar{q}_{n} .
$$

Let us assume that $x_{1} \in[0, a], x_{2} \in[0, b]$, where $a$ and $b$ are the length and the width of the plate, respectively.

Let us consider a sinusoidal load

$$
q_{n}=Q(t) \sin \frac{\pi x_{1}}{a} \sin \frac{\pi x_{2}}{b}
$$

and the boundary conditions (3). Then

$$
\bar{q}_{n}=\bar{Q}(s) \sin \frac{\pi x_{1}}{a} \sin \frac{\pi x_{2}}{b},
$$

and the solution of Eq. (54) is given by

$$
\bar{w}=\bar{w}_{\max }(s) \sin \frac{\pi x_{1}}{a} \sin \frac{\pi x_{2}}{b},
$$




\subsection{FGM plate}

Considering the symmetry of the material properties with respect to the mid-plane one gets a decoupling of the in-plane state and the plate state. Let us assume again the plate bending with $m=0$. Using [7] and the Laplace transform, one can reduce (1) to

$$
s \bar{D}_{\text {eff }} \Delta \Delta \bar{w}=\bar{q}_{n}-\frac{\bar{D}_{\text {eff }}}{\bar{\Gamma}} \Delta \bar{q}_{n}
$$

where $\bar{D}_{\text {eff }}=\bar{C}_{22}+\bar{C}_{33}$ is the Laplace transform of the effective bending stiffness relaxation function. Note that here $s \bar{D}_{\mathrm{eff}}=D_{\mathrm{eff}}^{0} \bar{\mu}(s)$, where $D_{\mathrm{eff}}^{0}=\left(C_{22}+C_{33}\right) / \mu(t)$.

To analyze the influence of the transverse shear stiffness on the deflection of the plate let us consider the bending of a rectangular plate made of an FGM. Using the assumption that $v=$ const and Eqs. (26), (27), and (49) are valid, we can rewrite Eq. (41) in the following form:

$$
s \bar{D}_{\mathrm{eff}} \Delta \Delta \bar{w}=\bar{q}_{n}-\frac{2}{\lambda^{2}(1-v)} \Delta \bar{q}_{n} .
$$

Introducing dimensionless variables by the formulae

$$
W=h^{-1} w, \quad X_{1}=h^{-1} x_{1}, \quad X_{2}=h^{-1} x_{2}, \quad X_{1} \in\left[0, \frac{a}{h}\right], \quad X_{2} \in\left[0, \frac{b}{h}\right],
$$

Eq. (58) transforms to

$$
s \bar{\mu}(s) \Delta \Delta \bar{W}=Q-\frac{2}{1-v} \frac{1}{\lambda^{2} h^{2}} \Delta Q .
$$

Here

$$
\Delta=\frac{\partial^{2}}{\partial X_{1}^{2}}+\frac{\partial^{2}}{\partial X_{2}^{2}}, \quad Q=\frac{\bar{q}_{n} h^{3}}{D_{\mathrm{eff}}^{0}} .
$$

Let us consider again a sinusoidal load (55) and the boundary conditions (3). Then the solution of Eq. (59) is given by

$$
\bar{W}=\frac{K}{\eta^{4} h^{4}} \frac{Q}{s \bar{\mu}(s)} \sin \frac{\pi h X_{1}}{a} \sin \frac{\pi h X_{2}}{b}, \quad K=1+\frac{2 \eta^{2}}{1-v} \frac{1}{\lambda^{2}} .
$$

For the Kirchhoff's plate theory $K=K_{K} \equiv 1$, for the homogeneous plate modeled in the sense of Mindlin's plate theory

$$
K=K_{M} \equiv 1+\frac{2 \eta}{1-v} \frac{1}{\pi^{2}} .
$$

Using bounds (34) for the FGM plate we obtain the inequalities

$$
1+\frac{2 \eta}{1-v} \frac{L^{2} m_{\min }^{2}}{\pi^{2} h^{2}} \leq K \leq 1+\frac{2 \eta}{1-v} \frac{L^{2} m_{\max }^{2}}{\pi^{2} h^{2}} .
$$

The influence of the shear stiffness on the deflection of the elastic FGM plate was given in [7]. For the viscoelastic plate both the qualitative and the quantitative influence of the shear stiffness is the same as in [7].

For example, let us consider an open-cell foam and the following values $v=0.3, a=b, h=0.05 a$, $\alpha=0.9$. Using the calculation of [7] we obtain the following values of $\lambda: \lambda=0.83 / h$ for $n=2, \lambda=0.82 / h$ for $n=5$. The corresponding values of factor $K$ are given by

$$
K_{\mathrm{M}} \approx 1.014, \quad K \approx 1.20 \quad(n=2), \quad K \approx 1.21 \quad(n=5) .
$$

That means that for the functionally graded plates the influence of transverse shear stiffness may be significant. As well as for elastic FGM plates for the cases of other types of boundary conditions the influence of the structure of the viscoelastic plate on the deflection may be greater than for the used simple support type boundary conditions. 


\section{Discussion and outlook}

The considered approach to model FGM plates within the framework of a five-parametric theory of plates has an advantage with respect to classical theories of sandwich or laminated plates. Further investigations should be directed to the more complex constitutive equations of viscoelastic solids taking into account thermomechanical behavior, impact processes and the description of the creep phenomenon in plates made of metal or polymer foams.

Acknowledgments The research work was partially supported by the Martin-Luther-University Halle-Wittenberg and the program of development of the South Federal University.

\section{References}

1. Altenbach, H., Zhilin, P.: A general theory of elastic simple shells (in Russian). Usp. Mek. 11, 107-14 (1988)

2. Altenbach, H.: Eine direkt formulierte lineare Theorie für viskoelastische Platten und Schalen. Ing. Arch. 58, 215-228 (1988)

3. Altenbach, H., Zhilin, P.: The theory of simple elastic shells. In: Kienzler, R., Altenbach, H., Ott, I. (eds.) Critical Review of the Theories of Plates and Shells and New Applications. Lect. Notes. Appl. Comp. Mech., vol. 16., pp. 1-12. Springer, Berlin (2004)

4. Altenbach, H.: Determination of elastic moduli of anisotropic plates with nonhomogeneous material in thickness direction (in Russian). Mech. Solids 22, 135-141 (1987)

5. Altenbach, H.: An alternative determination of transverse shear stiffnesses for sandwich and laminated plates. Int. J. Solids Struct. 37, 3503-3520 (2000)

6. Altenbach, H.: On the determination of transverse shear stiffnesses of orthotropic plates. ZAMP 51, 629-649 (2000)

7. Altenbach, H., Eremeyev, V.A.: Direct approach based analysis of plates composed of functionally graded materials. Arch. Appl. Mech. doi:10.1007/s00419-007-0192-3

8. Altenbach, H., Eremeyev, V.A.: Analysis of the viscoelastic behavior of plates made of functionally graded materials. ZAMM 88, 332-341 (2008)

9. Ashby, M.F., Evans, A.G., Fleck, N.A., Gibson, L.J., Hutchinson, J.W., Wadley, H.N.G.: Metal Foams: a Design Guide. Butterworth-Heinemann, Boston (2000)

10. Banhart, J., Ashby, M.F., Fleck, N.A. (eds.): Metal Foams and Porous Metal Structures. Verlag MIT Publishing, Bremen (1999)

11. Brinson, H.F., Brinson, C.L.: Polymer Engineering Science and Viscoelasticity. An Introduction. Springer, New York (2008)

12. Christensen, R.M.: Theory of Viscoelasticity. An Introduction. Academic Press, New York (1971)

13. Collatz, L.: Eigenwertaufgaben mit Technischen Anwendungen. Akademische Verlagsgesellschaft, Leipzig (1963)

14. Degischer, H.P., Kriszt, B. (eds.): Handbook of Cellular Metals. Production, Processing, Applications. Wiley-VCH, Weinheim (2002)

15. Drozdov, A.D.: Finite Elasticity and Viscoelasticity. World Scientific, Singapore (1996)

16. Gibson, L.J., Ashby, M.F.: Cellular Solids: Structure and Properties, 2nd edn. Cambridge Solid State Science Series. Cambridge University Press, Cambridge (1997)

17. Hartman, Ph.: Ordinary Differential Equations. Wiley, New York (1964)

18. Haupt, P.: Continuum Mechanics and Theory of Materials, 2nd edn. Springer, Berlin (2002)

19. Kraatz, A.: Berechnung des mechanischen Verhaltens von geschlossenzelligen Schaumstoffen unter Einbeziehung der Mikrostruktur. Diss., Zentrum für Ingenieurwissenschaften, Martin-Luther-Universität Halle-Wittenberg (2007)

20. Lakes, R.S.: Foam structures with a negative Poisson's ratio. Science 235, 1038-1040 (1987)

21. Lakes, R.S.: The time-dependent Poisson's ratio of viscoelastic materials can increase or decrease. Cell. Polym. 11, 466-469 (1992)

22. Lakes, R.S., Wineman, A.: On Poisson's ratio in linearly viscoelastic solids. J. Elast. 85, 45-63 (2006)

23. Landrock, A.H. (ed.): Handbook of Plastic Foams. Types, Properties, Manufacture and Applications. Noes Publications, Park Ridge (1995)

24. Lee, S.T., Ramesh, N.S. (eds.): Polymeric Foams. Mechanisms and Materials. CRC Press, Boca Raton (2004)

25. Mills, N.: Polymer Foams Handbook. Engineering and Biomechanics Applications and Design Guide. ButterworthHeinemann, Amsterdam (2007)

26. Mindlin, R.D.: Influence of rotatory inertia and shear on flexural motions of isotropic, elastic plates. Trans. ASME J. Appl. Mech. 18, 31-38 (1951)

27. Naghdi, P.M.: The theory of plates and shells. In: Flügge, S. (ed.) Handbuch der Physik, Bd. VIa/2, pp. 425-640. Springer, Berlin (1972)

28. Rabotnov Yu, N.: Elements of Hereditary Solid Mechanics. Mir Publishers, Moscow (1980)

29. Reissner, E.: On the theory of bending of elastic plates. J. Math. Phys. 23, 184-194 (1944)

30. Reissner, E.: The effect of transverse shear deformation on the bending of elastic plates. J. Appl. Mech. 12, A69-A77 (1945)

31. Reissner, E.: Reflection on the theory of elastic plates. Appl. Mech. Rev. 38, 1453-1464 (1985)

32. Riande, E. et al. (eds.): Polymer Viscoelasticity: Stress and Strain in Practice. Marcel Dekker, New York (2000)

33. Rothert, H.: Direkte Theorie von Linien- und Flächentragwerken bei viskoelastischem Werkstoffverhalten. Techn.-Wiss. Mitteilungen des Instituts für Konstruktiven Ingenieurbau 73-2. Ruhr-Universität, Bochum (1973)

34. Shaw, M.T., MacKnight, W.J.: Introduction to Polymer Viscoelasticity, 3rd edn. Wiley, Hoboken (2005)

35. Stoer, J., Bulirsch, R.: Introduction to Numerical Analysis. Springer, New York (1980) 
36. Timoshenko, S.P.: On the correnction for shear of the differential equation for transverse vibrations of prismatic bars. Philos. Mag. Ser. 6. 41, 744-746 (1921)

37. Tschoegl, N.W.: The Phenomenological Theory of Linear Viscoelastic Behavior. An Introduction. Springer, Berlin (1989)

38. Zhilin, P.A.: Applied Mechanics. Foundations of the Theory of Shells (in Russian). Petersburg State Polytechnical University, Saint Petersburg (2006)

39. Zhilin, P.A.: Mechanics of deformable directed surfaces. Int. J. Solids Struct. 12, 635-648 (1976) 